\title{
Il Fibroblast Growth Factor-23 (FGF-23)
}

\author{
D. Rendina, G. De Filippo, R. Muscariello, F. De Pascale, P. Strazzullo \\ Dipartimento di Medicina Clinica e Sperimentale, Università degli Studi di Napoli “Federico II", Napoli
}

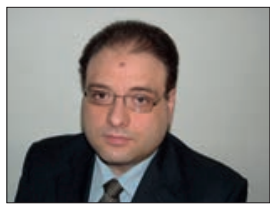

Domenico Rendina

\section{Introduzione}

Il fosforo (simbolo chimico P; peso atomico 15) è un costituente essenziale di DNA e RNA ed è coinvolto in numerosi processi biologici fondamentali per la vita dei vertebrati (1). Gli anioni fosfato sono presenti nel sangue in concentrazione pari a $0,75-1,45 \mathrm{mmol} / \mathrm{L}(2,32-4,49 \mathrm{mg} / \mathrm{dL})$ e in condizioni di sostanziale equilibrio con i fosfati intracellulari (2). I fosfati sono estremamente diffusi in natura e le loro principali fonti alimentari sono cereali, verdure, latte, carni bovine, pesce, pollame e legumi. Il fabbisogno alimentare medio di fosfati varia in funzione dell'età: nel primo anno di vita esso è pari a 300-600 mg/die mentre dai 25 anni in poi la dose raccomandata di fosfati è di $800 \mathrm{mg} /$ die (3). Il processo di assorbimento intestinale dei fosfati è estremamente efficace e avviene nel digiuno attraverso due meccanismi: uno passivo, sodio-indipendente, paracellulare e saturabile, che permette l'assorbimento del $70 \%$ dei fosfati assunti con la dieta; l'altro attivo, non saturabile e vitamina $\mathrm{D}$ - e sodio-dipendente $(2,3)$. Considerata la notevole disponibilità alimentare dei fosfati e l'elevata efficacia dei suoi processi di assorbimento digiunale, il controllo della omeostasi dei fosfati si realizza principalmente a livello del tubulo renale prossimale, dove due sistemi di trasporto sodio-dipendenti, i co-trasportatori sodio-fosfato di tipo IIa e IIc (NaPi-IIa o SLC34A1 e NaPi-IIc o SLC34A3), garantiscono il riassorbimento attivo di oltre il $90 \%$ del carico di fosfati filtrato a livello glomerulare $(4-6 \mathrm{gr} / \mathrm{die})(4,5)$.

Il Fibroblast Growth Factor-23 (FGF23; OMIM 605380) è stata la prima molecola di una nuova classe di ormoni denominati "fosfatonine" identificati per la proprietà di regolare il riassorbimento tubulare e l'escrezione urinaria dei fosfati (6, 7). Insieme al FGF15/19 e al FGF21, l'FGF23 appartiene al gruppo filogeneticamente più recente dei fattori di crescita per fibroblasti (FGFs), espresso esclusivamente nei vertebrati e dotato di attività endocrina (8).
Il ruolo fondamentale assunto dalla molecola nei processi di regolazione dell'omeostasi dei fosfati è stato recentemente confermato da un ampio studio di epidemiologia genetica condotto con il metodo GWAS (Genome-Wide Association Study) (9).

\section{FGF23: Proprietà biologiche}

FGF23 è una glicoproteina codificata dall'omonimo gene (FGF23) localizzato sul cromosoma 12, nella regione 12 p13.3 (http://www.ncbi.nlm.nih.gov/omim/605380), prodotta principalmente dagli osteociti. Liniziale trascritto genico, costituito da una proteina di 251 aminoacidi (30 $\mathrm{kDa}$ ), contiene una sequenza-segnale di 24 aminoacidi che non è presente nella molecola secreta dosabile nel siero e nel plasma (7, 10-12) (Fig.1).

FGF23 esercita le proprie attività biologiche attraverso l'interazione con un recettore di membrana (FGFR) di cui sono descritti 4 sottotipi, indicati con i numeri arabi da 1 a $4(8,13,14)$. Questi ultimi sono comuni a tutti i componenti della famiglia FGFs e sono caratterizzati dalla presenza di tre domini extracellulari strutturalmente simili alle immunoglobuline $(8,13,14)$. I dati attualmente disponibili in letteratura evidenziano che FGF23 interagisce con le isoforme 1, 3 e 4 di FGFR $(15,16)$. A livello tissutale le attività endocrine di FGF23 sono rese specifiche dall'interazione del complesso FGF23-FGFR con Klotho (17-19). Klotho è una molecola di 1014 aminoacidi con un singolo dominio extracellulare e una corta coda intracellulare (proteina di membrana di tipo 1): la mancata espressione di Klotho induce in modelli sperimentali un fenotipo identico a quello indotto dalla mancata espressione di FGF23 $(18,19)$. Yamazaki et al, utilizzando specifici anticorpi monoclonali, hanno recentemente dimostrato che i domini N- e C-terminali di FGF23 sono rispettivamente responsabili dell'interazione con FGFR e Klotho e che entrambe le interazioni sono indispensabili per l'attività biologica di 


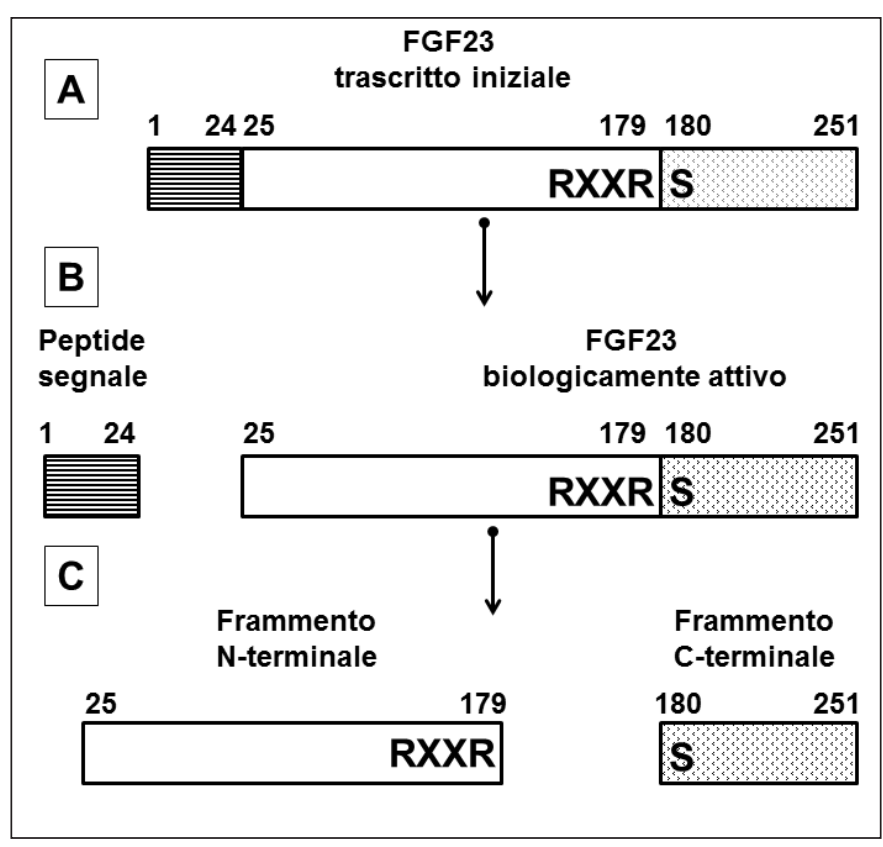

Figura 1 - Struttura della proteina FGF23.

Il trascritto iniziale della proteina FGF23 è costituito da 215 aminoacidi con una sequenza segnale di 24 aminoacidi (A) che viene staccata prima della secrezione della molecola biologicamente attiva (B). La degradazione enzimatica della proteina avviene attraverso un clivaggio tra gli aminoacidi arginina 179 e serina180 ad opera di specifici enzimi proteolitici che riconoscono la sequenza arginina176-X-X-arginina179 e determinano la produzione dei due frammenti $\mathrm{N}$ - e $\mathrm{C}$ - terminale (C).

\section{FGF23 (20).}

Il principale sito d'azione di FGF23 è il tubulo renale prossimale $(7,21)$. A questo livello, come schematizzato nella Figura 2, FGF23 esercita il proprio controllo sull'omeostasi dei fosfati sia regolando l'espressione cellulare di NaPi-IIa e NaPi-IIc sia riducendo la biosintesi della forma attiva della vitamina $\mathrm{D}$, la $1,25(\mathrm{OH})_{2} \mathrm{D}_{3}(21)$. La regolazione dell'espressione cellulare delle due isoforme di NaPi-II avviene principalmente attraverso l'interazione con FGFR1 (15). Linibizione della biosintesi di $1,25(\mathrm{OH})_{2} \mathrm{D}_{3}$ avviene attraverso l'interazione di FGF23 con le isoforme 3 e 4 di FGFR e si realizza tramite una inibizione funzionale dell'enzima 1alfa-idrossilasi e una stimolazione funzionale dell'enzima 25(OH)D-24-hydroxylase (16). Alla ridotta biosintesi renale di $1,25(\mathrm{OH})_{2} \mathrm{D}_{3}$ segue la riduzione dell'assorbimento intestinale (digiunale) dei fosfati (22). Le attività biologiche di FGF23 a livello del tubulo renale sembrano indipendenti dalle concentrazioni seriche del PTH (8).

Dati sperimentali preliminari indicano che anche il tessuto paratiroideo e quello osseo sono potenziali siti d'azione di FGF23, con meccanismi biologici e conseguenze fun-

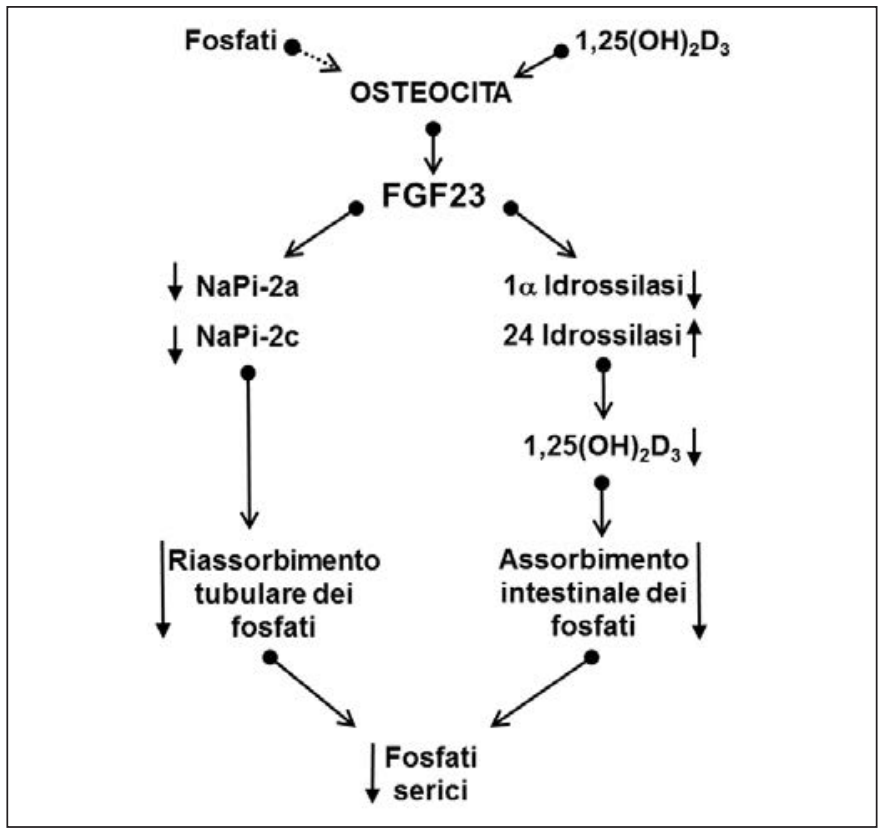

Figura 2 - Meccanismo d'azione di FGF23.

zionali in corso di definizione (23-25).

La molecola proteica intatta di FGF23 è dosabile nel sangue ed è dotata di piena attività biologica: la sua inattivazione avviene attraverso un clivaggio tra gli aminoacidi arginina179 e serina180 mediato da specifici enzimi proteolitici che riconoscono la sequenza arginina176-X-X-arginina179 (21). Lo stato di O-glicosilazione della molecola di FGF23 influenza in maniera significativa il processo di degradazione enzimatica della stessa, in quanto la molecola completamente glicosilata (3 siti) è resistente al processo di clivaggio, mentre la molecola con uno o due siti glicosilati viene degradata (26). Dal clivaggio di FGF23 risulta un frammento C-terminale di $12 \mathrm{kDa}$ e uno $\mathrm{N}$-terminale di $18 \mathrm{kDa}$ (21). Non esistono al momento dati univoci riguardanti l'attività biologica di questi frammenti, che secondo alcuni dati sperimentali manterrebbero una propria attività biologica fosfaturica e richiederebbero per l'inattivazione una successiva degradazione enzimatica (27).

I dati al momento disponibili in letteratura indicano l'esistenza di un meccanismo di feedback negativo tra la biosintesi ossea di FGF23 e i livelli circolanti di $1,25(\mathrm{OH})_{2} \mathrm{D}_{3}$ (28); non sembrano attualmente conclusivi quelli riguardanti gli effetti a breve e lungo termine dell'apporto dietetico di fosfati sui livelli circolanti di FGF23 (29-31).

Lidentificazione di FGF23 e delle sue proprietà biologiche è avvenuta grazie alle osservazioni eseguite in pazienti affetti da rari disordini congeniti (Autosomal-Dominant Hypophosphatemic Rickets ADHR, OMIM 193100) o acquisiti (osteomalacia oncogenica) dell'omeostasi dei fosfati, caratterizza- 
ti da una ridotta soglia massima di riassorbimento tubulare dei fosfati in base al nomogramma di Walton e Bijvoet (11, $32,33)$. La perdita renale di fosfati secondaria alla ridotta soglia di riassorbimento tubulare di fosfati determina una severa ipofosfatemia con calcemia e paratormone normali e livelli circolanti di $1,25(\mathrm{OH})_{2} \mathrm{D}_{3}$ inappropriatamente normali in relazione ai livelli ematici dei fosfati (7). Nella maggioranza dei soggetti affetti da queste patologie e da altre con simili caratteristiche fenotipiche, i livelli circolanti di FGF23 sono significativamente superiori (anche di due ordini di grandezza) a quelli rilevabili nei soggetti sani (34-36).

E necessario tuttavia rilevare che una condizione clinica di perdita renale di fosfati e fosfatemia inferiore a $2,5 \mathrm{mg} /$ $\mathrm{dL}$, in presenza di normale funzionalità paratiroidea, si riscontra anche in alcuni soggetti portatori di condizioni estremamente comuni nella popolazione generale quali la nefrolitiasi calcica recidivante e l'osteoporosi idiopatica $(35,37,38)$. In particolare, i soggetti affetti da calcolosi renale recidivante con perdita renale di fosfati presentano in media livelli circolanti di FGF23 significativamente superiori rispetto ai soggetti calcolotici senza perdita renale di fosfato e ai soggetti sani, anche se questi livelli sono sensibilmente inferiori a quelli osservati nel rachitismo ipofosfatemico e nell'osteomalacia oncogenica (35).

Oltre che in patologie caratterizzate fenotipicamente dalla perdita renale di fosfati, elevati livelli circolanti di FGF23 sono stati riscontrati in pazienti con insufficienza renale cronica, nei quali essi potrebbero concorrere alla patogenesi delliperparatiroidismo secondario e terziario e alla definizione del rischio cardiovascolare globale, nonché in pazienti con disordini paratiroidei (39-43). Il ruolo di FGF23 (e delle altre fosfatonine) nella patogenesi dei sud-

TABELLA I - RUOLO DI FGF23 E DELLE ALTRE FOSFATONINE IN DIVERSI DISORDINI CLINICI

\begin{tabular}{|c|c|c|}
\hline & Caratteristiche fenotipiche & Commenti \\
\hline $\mathrm{ADHR}$ & \multirow{3}{*}{$\begin{array}{l}\text { Perdita renale di fosfati, ipofosfatemia, } \\
\text { livelli di } 1,25(\mathrm{OH})_{2} \mathrm{D}_{3} \text { inappropriatamente normali, } \\
\text { rachitismo }\end{array}$} & \multirow{3}{*}{$\begin{array}{l}\text { Elevati livelli ematici di FGF23 ed altre fosfatonine (FGF7, } \\
\text { sFRP-4, MEPE) in associazione a mutazioni strutturali dei } \\
\text { geni } F G F 23 \text { (ADHR), PHEX (XLHR forma dominante), } \\
D M P 1 \text { (ARHR1), ENPP1 (ARHR2) e CLCN5 (XLHR } \\
\text { forma recessiva) }\end{array}$} \\
\hline $\begin{array}{l}\text { XLHR forma dominante } \\
\text { ARHR1 } \\
\text { ARHR2 }\end{array}$ & & \\
\hline XLHR forma recessiva & & \\
\hline Osteomalacia oncogenica & $\begin{array}{l}\text { Perdita renale di fosfati, ipofosfatemia, } \\
\text { livelli di } 1,25(\mathrm{OH})_{2} \mathrm{D}_{3} \text { inappropriatamente normali, } \\
\text { osteomalacia }\end{array}$ & $\begin{array}{l}\text { Iper-produzione di fosfatonine } \\
\text { di origine neoplastica (PMTMCT, Phosphaturic } \\
\text { Mesenchimal Tumor Mixed Connective Tissue) }\end{array}$ \\
\hline $\begin{array}{l}\text { Osteodisplasia fibrosa di } \\
\text { McCune-Albright }\end{array}$ & $\begin{array}{l}\text { Displasia fibrosa ossea, patologie endocrine e lesioni } \\
\text { cutanee pigmentate. In oltre } \\
\text { la metà dei pazienti è presente } \\
\text { un disordine da perdita renale } \\
\text { di fosfati }\end{array}$ & $\begin{array}{l}\text { Elevati livelli ematici di FGF23 } \\
\text { per iper-produzione della molecola } \\
\text { a livello del tessuto osseo patologico }\end{array}$ \\
\hline $\begin{array}{l}\text { Sindrome del nevo sebaceo } \\
\text { lineare }\end{array}$ & $\begin{array}{l}\text { Nevi sebacei, anomalie sistema nervoso centrale, oculari } \\
\text { e scheletriche. In alcuni casi, è presente una forma di } \\
\text { rachitismo ipofosforemico ad esordio neonatale }\end{array}$ & $\begin{array}{l}\text { Elevati livelli ematici di FGF23 nei soggetti con rachitismo } \\
\text { ipofosforemico }\end{array}$ \\
\hline Nanismo osteoglofonico & $\begin{array}{l}\text { Bassa statura, craniosinostosi, adentulia multipla, } \\
\text { perdita renale di fosfati }\end{array}$ & $\begin{array}{l}\text { Elevati livelli ematici di FGF23 } \\
\text { in associazione a mutazioni missense } \\
\text { del gene FGFR1 }\end{array}$ \\
\hline $\begin{array}{l}\text { Nefrolitiasi renale recidivante } \\
\text { Osteoporosi primitiva }\end{array}$ & $\begin{array}{l}\text { Perdita renale di fosfati con ipofosfatemia in una } \\
\text { percentuale significativa dei pazienti }\end{array}$ & $\begin{array}{l}\text { Livelli ematici di FGF23 aumentati o inappropriatamente } \\
\text { normali in relazione all'ipofosforemia }\end{array}$ \\
\hline Ipoparatiroidismo & $\begin{array}{l}\text { Ipocalcemia, iperfosfatemia, normomagnesemia, PTH } \\
\text { non rilevabile }\end{array}$ & $\begin{array}{l}\text { Elevati livelli serici di fosfati determinano un aumento dei } \\
\text { livelli ematici di FGF23 }\end{array}$ \\
\hline Iperparatiroidismo & $\begin{array}{l}\text { Ipercalcemia, ipofosfatemia, normomagnesemia, } \\
\text { iperfosfaturia, livelli di PTH inappropriati in relazione } \\
\text { all'ipocalcemia }\end{array}$ & $\begin{array}{l}\text { Elevati livelli ematici di FGF23 sono stati rilevati in } \\
\text { pazienti con iperparatiroidismo primario }\end{array}$ \\
\hline Insufficienza renale cronica & $\begin{array}{l}\text { Riduzione permanente e progressiva della funzione } \\
\text { renale per danno irreversibile } \\
\text { al parenchima renale }\end{array}$ & $\begin{array}{l}\text { Elevati livelli serici di fosfati e/o una ridotta clearance di } \\
\text { FGF23 o dei suoi frammenti determinano un aumento } \\
\text { dei livelli ematici di FGF23 }\end{array}$ \\
\hline $\begin{array}{l}\text { Ipofosforemia post-trapianto } \\
\text { renale }\end{array}$ & $\begin{array}{l}\text { Persistente ipofosfatemia non associata ad elevati livelli } \\
\text { serici di PTH nel decorso post-operatorio }\end{array}$ & Elevati livelli ematici di FGF23 \\
\hline Calcinosi tumorale & $\begin{array}{l}\text { Iperfosfatemia, ipofosfaturia, livelli di } 1,25(\mathrm{OH})_{2} \mathrm{D}_{3} \\
\text { elevati o normali, calcificazioni ectopiche }\end{array}$ & $\begin{array}{l}\text { Mutazioni dei geni } F G F 23, G A L N T 3 \\
\text { e Klotho causanti una riduzione dell'attività biologica di } \\
\text { FGF23 }\end{array}$ \\
\hline
\end{tabular}

ADHR Rachitismo ipofosforemico autosomico dominante; XLH: Rachitismo ipofosforemico X-linked; ARHR Rachitismo ipofosforemico autosomico recessivo. 
detti disordini clinici è schematizzato nella Tabella I. In questo scenario, la recente identificazione delle proprietà biologiche delle regioni C-terminale e N-terminale di FGF23, responsabili rispettivamente dellinterazione con FGFR e Klotho, potrebbe aprire nuove prospettive terapeutiche nel medio-breve termine (20). In particolare, rivestono interesse le potenziali applicazioni terapeutiche legate allo sviluppo di anticorpi monoclonali anti-FGF23 e di molecole ricombinanti di FGF23 (44-46). La somministrazione di anticorpi neutralizzanti FGF23 in topi Hyp, il modello animale delle forme di rachitismo ipofosfatemico, ha determinato la normalizzazione in un tempo relativamente breve (circa 4 settimane) dei livelli serici di fosfati e di $1,25(\mathrm{OH})_{2} \mathrm{D}_{3}$ e un significativo miglioramento delle deformazioni ossee $(44,45)$. Anche la somministrazione del solo frammento C-terminale della molecola di FGF23 determina una riduzione dell'escrezione renale di fosfato con aumento dei livelli circolanti di fosfati sia in topi sani sia in topi Hyp. In questo caso il meccanismo farmacodinamico proposto è quello dell'inibizione recettoriale dell'azione dell'FGF23: il frammento C-terminale dell'FGF23 occuperebbe il sito di legame della molecola nativa su FGFR ma non sarebbe in grado di attivare il complesso FGFR-Klotho per la mancanza dell'estremità N-terminale della molecola (46). Oltre che nei soggetti con rachitismo ipofosfatemico, la somministrazione di anticorpi anti-FGF23 o di frammenti con affinità inibitoria per il recettore ma senza attività biologica potrebbe trovare un'indicazione terapeutica anche nei pazienti con osteomalacia oncogenica, nei casi in cui il tumore causale sia non identificato o non aggredibile chirurgicamente, e in generale nei soggetti con condizioni cliniche caratterizzate da ipofosforemia associata ad elevati livelli di FGF23.

Nei soggetti con forme iniziali di insufficienza renale cronica, è ipotizzabile in linea teorica l'utilizzo di FGF23 ricombinante al fine di aumentare l'escrezione renale di fosfati e migliorare il rapporto calcio/fosfati, anche se l'inibizione dell'espressione cellulare di 1alfa idrossilasi potrebbe facilitare l'insorgenza di iperparatiroidismo secondario. Molto interessante appare l'utilizzo di FGF23 ricombinante nei soggetti con iperfosfatemia e calcinosi tumorale, una rara malattia determinata da mutazioni dei geni FGF23, GALNT3 (UDP-N-acetyl-alpha-Dgalactosamine:polypeptide N-acetylgalactosaminyltransferase 3) e Klotho, caratterizzata dal riscontro di bassi livelli circolanti di FGF23 integro biologicamente attivo (http://www.ncbi.nlm.nih.gov/omim/211900).

\section{Conclusioni}

Lidentificazione delle proprietà biologiche di FGF23 ha determinato una rivoluzione copernicana nella com- prensione dei meccanismi fisiologici che regolano l'omeostasi dei fosfati e il metabolismo della vitamina D. Il sospetto clinico di un disordine da eccessiva attività biologica di FGF23 deve fondarsi sul riscontro di bassi livelli ematici di fosfati (inferiori a $4 \mathrm{mg} / \mathrm{dL}$ nei neonati, $3,5 \mathrm{mg} / \mathrm{dL}$ nei bambini e $2,5 \mathrm{mg} / \mathrm{dL}$ negli adulti) in soggetti con normale funzione paratiroidea (normocalcemia e normali livelli circolanti di PTH). A questo punto, come indagini di secondo livello, è opportuno valutare i livelli circolanti di $1,25(\mathrm{OH})_{2} \mathrm{D}_{3}, 25 \mathrm{OHD}_{3}$ e stimare la soglia di riassorbimento tubulare dei fosfati, che viene determinata in base ai livelli serici e urinari di fosfati e creatinina (47). È opinione condivisa nel mondo scientifico internazionale che il riscontro di valori plasmatici dosabili di FGF23 in una condizione clinica fenotipicamente caratterizzata da perdita renale di fosfati sia indicativo di un'eccessiva azione biologica di FGF23 (34, $35,48)$. Oltre che in pazienti con disordini relativamente rari da perdita renale di fosfati, FGF23 svolge un ruolo patogenetico fondamentale in alterazioni comuni quali l'insufficienza renale e i disordini paratiroidei. In tutte queste patologie, l'identificazione delle proprietà biologiche delle regioni C-terminale e N-terminale di FGF23 sta aprendo interessanti prospettive terapeutiche nel medio-breve termine.

\section{Riassunto}

Negli ultimi due decenni, l'identificazione delle proprietà biologiche del Fibroblast Growth Factor 23 (FGF23) ha notevolmente ampliato le conoscenze sulla regolazione endocrina e paracrina dell'omeostasi dei fosfati e sulle relazioni intercorrenti tra asse rene-intestino-tessuto osseo e sistema biologico vitamina D-PTH-calcio-fosfati. Lo studio delle proprietà biologiche di FGF23 ha inoltre aperto interessanti campi di ricerca in ambito clinico e farmacologico.

Parole chiave: Fibroblast Growth Factor 23, Omeostasi dei fosfati, Vitamina D, PTH

\section{Indirizzo degli Autori:}

Domenico Rendina, MD

Dipartimento di Medicina Clinica e Sperimentale

Università degli Studi di Napoli "Federico II"

via Sergio Pansini 5

80131- Napoli

domenico.rendina@unina.it 


\section{Bibliografia}

1. Bringhurst FR. Regulation of calcium and phosphate homeostasis. In: DeGroot LJ, Jameson JL, eds. Endocrinology, Philadelphia: W.B. Saunders Co. 2001; 1029-52.

2. Takeda E, Taketani Y, Sawada N, Sato T, Yamamoto H. The regulation and function of phosphate in the human body. Biofactors 2004; 21: 345-55.

3. Takeda E, Yamamoto H, Nashiki K, Sato T, Arai H, Taketani Y. Inorganic phosphate homeostasis and the role of dietary phosphorus. J Cell Mol Med 2004; 8: 191-200.

4. Forster IC, Hernando N, Biber J, Murer H. Proximal tubular handling of phosphate: a molecular perspective. Kidney Int 2006; 70: 1548-59.

5. Miyamoto K, Ito M, Tatsumi S, Kuwahata M, Segawa H. New aspect of renal phosphate reabsorption: the type IIc sodium-dependent phosphate transporter. Am J Nephrol 2007; 27: 503-15.

6. Econs MJ, Drezner MK. Tumor-induced osteomalaciaunveiling a new hormone. N Engl J Med 1994; 330: 167981.

7. Fukumoto S, Yamashita T. FGF23 is a hormone-regulating phosphate metabolism-unique biological characteristics of FGF23. Bone 2007; 40: 1190-5.

8. Itoh N. Hormone-like (endocrine) Fgfs: their evolutionary history and roles in development, metabolism, and disease. Cell Tissue Res 2010; 342: 1-11.

9. Kestenbaum B, Glazer NL, Köttgen A, et al. Common genetic variants associate with serum phosphorus concentration. J Am Soc Nephrol 2010; 21: 1223-32

10. Mirams M, Robinson BG, Mason RS, Nelson AE. Bone as a source of FGF23: regulation by phosphate? Bone 2004; 35: 1192-9.

11. Shimada T, Mizutani S, Muto T, et al. Cloning and characterization of FGF23 as a causative factor of tumor induced osteomalacia. Proc Natl Acad Sci USA 2001; 98: 6500-5.

12. Weber TJ, Liu S, Indridason OS, Quarles LD. Serum FGF23 levels in normal and disordered phosphorus homeostasis. J Bone Miner Res 2003; 18: 1227-34.

13. Werner S, Duan DS, de Vries C, Peters KG, Johnson DE, Williams LT. Differential splicing in the extracellular region of fibroblast growth factor receptor 1 generates receptor variants with different ligand-binding specificities. Mol Cell Biol 1992; 12: 82-8.

14. Itoh N, Ornitz DM. Evolution of the Fgf and Fgfr gene families. Trends Genet 2004; 20: 563-9.

15. Gattineni J, Bates C, Twombley K, et al. FGF23 decreases renal NaPi-2a and NaPi-2c expression and induces hypophosphatemia in vivo predominantly via FGF receptor 1. Am J Physiol Renal Physiol 2009; 297: F282-91.

16. Gattineni J, Twombley K, Goetz R, Mohammadi M, Baum
M. Regulation of Serum 1,25(OH)2Vitamin D3 levels by Fibroblast Growth Factor 23 is Mediated by FGF Receptors 3 and 4. Am J Physiol Renal Physiol 2011, in press

17. Urakawa I, Yamazaki Y, Shimada T, et al. Klotho converts canonical FGF receptor into a specific receptor for FGF23. Nature 2006; 444: 770-4.

18. Kurosu H, Ogawa Y, Miyoshi M, et al. Regulation of fibroblast growth factor-23 signaling by klotho. J Biol Chem 2006; 281: 6120-3.

19. Razzaque MS, Lanske B. The emerging role of the fibroblast growth factor-23-klotho axis in renal regulation of phosphate homeostasis. J Endocrinol 2007; 194: 1-10

20. Yamazaki Y, Tamada T, Kasai N, et al. Anti-FGF23 neutralizing antibodies demonstrate the physiological role and structural features of FGF23. J Bone Miner Res 2008; 23: 1509-18.

21. Yu X, Ibrahimi OA, Goetz R, et al. Analysis of the biochemical mechanisms for the endocrine actions of fibroblast growth factor-23. Endocrinology 2005; 146: 4647-56.

22. Jüppner H. Phosphate and FGF-23. Kidney Int Suppl 2011; (121): S24-7.

23. Lavi-Moshayoff V, Wasserman G, Meir T, Silver J, NavehMany T. PTH increases FGF23 gene expression and mediates the high-FGF23 levels of experimental kidney failure: a bone parathyroid feedback loop. Am J Physiol Renal Physiol 2010; 299: F882-9.

24. Silver J, Naveh-Many T. FGF23 and the parathyroid glands. Pediatr Nephrol 2010; 25(11): 2241-5.

25. Shalhoub V, Ward SC, Sun B, et al. Fibroblast Growth Factor 23 (FGF23) and Alpha-Klotho Stimulate Osteoblastic MC3T3.E1 Cell Proliferation and Inhibit Mineralization. Calcif Tissue Int 2011; in press.

26. Frishberg Y, Ito N, Rinat C, et al. Hyperostosis-hyperphosphatemia syndrome: a congenital disorder of O-glycosylation associated with augmented processing of fibroblast growth factor 23. J Bone Miner Res 2007; 22: 235-42.

27. Berndt TJ, Craig TA, McCormick DJ, et al. Biological activity of FGF-23 fragments. Pflugers Arch 2007; 454: 615-23.

28. Kolek OI, Hines ER, Jones MD, et al. 1alpha, 25-Dihydroxyvitamin D3 upregulates FGF23 gene expression in bone: the final link in a renal-gastrointestinalskeletal axis that controls phosphate transport. Am J Physiol Gastrointest Liver Physiol 2005; 289: G1036-42.

29. Ferrari SL, Bonjour JP, Rizzoli R. Fibroblast growth factor-23 relationship to dietary phosphate and renal phosphate handling in healthy young men. J Clin Endocrinol Metab 2005; 90: 519-24.

30. Antoniucci DM, Yamashita T, Portale AA. Dietary phosphorus regulates serum fibroblast growth factor-23 concentrations in healthy men. J Clin Endocrinol Metab 2006; 91: 3144-9.

31. Vervloet MG, van Ittersum FJ, Büttler RM, Heijboer AC, 
Blankenstein MA, ter Wee PM. Effects of dietary phosphate and calcium intake on fibroblast growth factor-23. Clin J Am Soc Nephrol 2011; 6: 383-9.

32. ADHR Consortium. Autosomal dominant hypophosphataemic rickets is associated with mutations in FGF23. Nat Genet 2000; 26: 345-8.

33. Walton RJ, Bijvoet OL. Nomogram for derivation of renal threshold phosphate concentration. Lancet 1975; 2: 309-10.

34. Endo I, Fukumoto S, Ozono K, et al. Clinical usefulness of measurement of fibroblast growth factor 23 (FGF23) in hypophosphatemic patients: proposal of diagnostic criteria using FGF23 measurement. Bone 2008; 42: 1235-9.

35. Rendina D, Mossetti G, De Filippo G, Cioffi M, Strazzullo P. Fibroblast growth factor 23 is increased in calcium nephrolithiasis with hypophosphatemia and renal phosphate leak. J Clin Endocrinol Metab 2006; 91: 959-63.

36. Rendina D, De Filippo G, Tauchmanovà L, et al. Bone turnover and the osteoprotegerin-RANKL pathway in tumorinduced osteomalacia: a longitudinal study of five cases. Calcif Tissue Int 2009; 85: 293-300.

37. Prié D, Huart V, Bakouh N, et al. Nephrolithiasis and osteoporosis associated with hypophosphatemia caused by mutations in the type 2 a sodium-phosphate cotransporter. N Engl J Med 2002; 347: 983-91.

38. Prié D, Ravery V, Boccon-Gibod L, Friedlander G. Frequency of renal phosphate leak among patients with calcium nephrolithiasis. Kidney Int 2001; 60: 272-6.

39. Isakova Evenepoel P, Meijers B, Viaene L, et al. Fibroblast growth factor-23 in early chronic kidney disease: additional support in favor of a phosphate-centric paradigm for the pathogenesis of secondary hyperparathyroidism. Clin J Am Soc Nephrol 2010; 5: 1268-76.
40. Isakova T, Wahl P, Vargas GS, et al. Fibroblast growth factor 23 is elevated before parathyroid hormone and phosphate in chronic kidney disease. Kidney Int 2011; in press.

41. Yamashita H, Yamazaki Y, Hasegawa H, et al. Fibroblast growth factor-23 (FGF23) in patients with transient hypoparathyroidism: its important role in serum phosphate regulation. Endocr J 2007; 54: 465-70.

42. Kobayashi K, Imanishi Y, Miyauchi A, et al. Regulation of plasma fibroblast growth factor 23 by calcium in primary hyperparathyroidism. Eur J Endocrinol 2006; 154: 93-9.

43. Gupta A, Winer K, Econs MJ, Marx SJ, Collins MT. FGF23 is elevated by chronic hyperphosphatemia. J Clin Endocrinol Metab 2004; 89: 4489-92.

44. Aono Y, Yamazaki Y, Yasutake J, et al. Therapeutic effects of anti-FGF23 antibodies in hypophosphatemic rickets/ osteomalacia. J Bone Miner Res 2009; 24: 1879-88.

45. Aono Y, Hasegawa H, Yamazaki Y, et al. Anti-FGF-23 neutralizing antibodies ameliorate muscle weakness and decreased spontaneous movement of Hyp mice. J Bone Miner Res 2011; 26: 803-10.

46. Goetz R, Nakada Y, Hu MC, et al. Isolated C-terminal tail of FGF23 alleviates hypophosphatemia by inhibiting FGF23-FGFR-Klotho complex formation. Proc Natl Acad Sci USA 2010; 107: 407-12.

47. Bastepe M, Jüppner H. Inherited hypophosphatemic disorders in children and the evolving mechanisms of phosphate regulation. Rev Endocr Metab Disord 2008; 9: 171-80.

48. Rendina D, De Filippo G, Strazzullo P. NHERF1 mutations and responsiveness of renal parathyroid hormone. N Engl J Med 2008; 359: 2616-7.

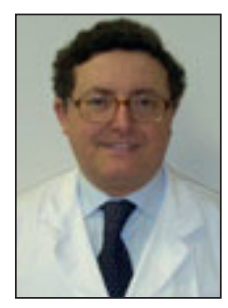

L'Editor ricorda il compianto

Giuseppe Mossetti

amico e collega stimato 